\title{
Causality in Relativistic Many Body Theory*
}

\author{
H. Blum and R. Brockmann \\ Institut für Kernphysik, Johannes Gutenberg-Universität, D-55099 Mainz, Germany
}

\begin{abstract}
The stability of the nuclear matter system with respect to density fluctuations is examined exploring in detail the pole structure of the electro-nuclear response functions. Making extensive use of the method of dispersion integrals we calculate the full polarization propagator not only for real energies in the spacelike and timelike regime but also in the whole complex energy plane. The latter proved to be necessary in order to identify unphysical causality violating poles which are the consequence of a neglection of vacuum polarization. On the contrary it is shown that Dirac sea effects stabilize the nuclear matter system shifting the unphysical pole from the upper energy plane back to the real axis. The exchange of strength between these real timelike collective excitations and the spacelike energy regime is shown to lead to a reduction of the quasielastic peak as it is seen in electron scattering experiments. Neglecting vacuum polarization one also obtains a reduction of the quasielastic peak but in this case the strength is partly shifted to the causality violating pole mentioned above which consequently cannot be considered as a physical reliable result. Our investigation of the response function in the energy region above the threshold of nucleon anti-nucleon production leads to another remarkable result. Treating the nucleons as point-like Dirac particles we show that for any isospin independent NN-interaction RPA-correlations provide a reduction of the production amplitude for $\mathrm{p} \overline{\mathrm{p}}$-pairs by a factor 2 .
\end{abstract}

\section{INTRODUCTION}

Dirac sea effects are perhaps the most interesting characteristic of relativistic many body theory. In nuclear matter e.g. the effective mass of the nucleons and their coupling to the mesons undergo considerable corrections due to vacuum fluctuations [1], [2]. In addition, relativistic many body theory predicts strong corrections of the properties of hadrons at high nuclear matter densities. Unfortunately, up to now it proved to be very difficult to find unambiguous experimental evidence for such effects. One method to probe the Dirac sea is to investigate the production of antiprotons and other antibaryons in heavy ion collisions. Such experiments are currently performed by several groups under quite different kinematic conditions. These range from subthreshold production of antiprotons in $\mathrm{Ni}+\mathrm{Ni}$ collisions at $1.85 \mathrm{GeV} /$ nucleon at SIS (GSI) [3] up to antibaryon production in Au+Au collisions at 14.6 $\mathrm{GeV} /$ nucleon at AGS (E878 Collaboration at BNL) [4 and sulphur-nucleus collisions at $200 \mathrm{GeV} / \mathrm{nucleon}$ at SPS (NA35 collaboration at Cern) [5]. Beside the search for evidence of the quark-gluon plasma [6] the attention is drawn to possible changes of the properties of the nucleons and especially the vector mesons [7] - 90 in the nuclear medium.

The presence of a filled Dirac sea of antiparticles, in addition to the Fermi sea of protons and neutrons, has consequences not only for the static properties mentioned above, but also for the dynamics of nuclear matter. The latter may be probed e.g. by measuring the response of the system to the disturbance induced by an external virtual photon, as it is done in inelastic electron scattering experiments. A well known and as yet unsolved problem in this field is an apparent quenching (in comparison with the prediction of simple one-particle models) of the longitudinal contribution to the inclusive scattering cross section, particularly for medium heavy and heavier nuclei like ${ }^{40} \mathrm{Ca}$, ${ }^{56} \mathrm{Fe}$ and ${ }^{238} \mathrm{U}$. First observed for momentum transfers of some $100 \mathrm{MeV} / \mathrm{c}$ [10], [1], a SLAC experiment [12] later showed that the quenching even persists up to $1 \mathrm{GeV} / \mathrm{c}$. This may be taken as a hint that relativistic effects play a role. Further, it seems reasonable that in heavier nuclei collective phenomena reduce the strength of longitudinal one-particle excitations. So the quenching problem of the longitudinal response function appears as an appropriate subject for the application of relativistic many body theory.

The first conserving approximation which also includes collective phenomena in a proper way is the random phase approximation (RPA). RPA-calculations of the response functions for inelastic electron scattering have been done by several authors [13] - [18]. In the framework of Walecka's $\sigma \omega$-model [1] the first calculations were performed for nuclear matter [13] - [15] and then applied to finite nuclei via the local density approximation (LDA) [16], [17]. As a

\footnotetext{
${ }^{*}$ Supported by the Deutsche Forschungsgemeinschaft (SFB 201)
} 
result of many body correlations, in RPA the longitudinal response function is reduced by about 10-20\% compared to the Mean Field (MFA) and Hartree approximation (HA). This reproduces at least qualitatively the trend of the experimental data. Similar results were obtained in a finite nucleus calculation [18].

Despite the final curves of [13] - 18 seem to confirm the same qualitative picture, they are obtained from two completely different approximation schemes. While vacuum polarization was neglected in [13] and [16], it is included in the other calculations. In view of the results for the response functions one could argue that these seem not to be very sensitive with respect to Dirac sea effects. But this is not the case. The true situation is veiled by the fact that all calculations were performed only for the space-like kinematic region $\left(q^{2}=q_{0}^{2}-\vec{q}^{2}<0\right)$. The reason therefore is of course that only this regime is accessible by electron scattering.

The aim of the present paper now is to draw attention to the fact, that effects in the space- and time-like energy regimes, which seem to be so well separated in view of the completely different experimental methods of investigation sketched above, nevertheless have an influence on each other. Especially it is our goal to show, that calculations which are performed to explain experimental data in one of the two kinematic regions have at least to be checked, whether for the other regime these provide results, which are physically consistent at least within the model used. So it will be shown that in the $\sigma \omega$-model only calculations including Dirac sea effects will preserve causality.

However, this becomes obvious only if one looks at the time-like part of the electro-nuclear response function. In RPA one would expect to find there a sharp peak corresponding to the $\sigma$ - and the $\omega$-meson respectively, which are the transmitter of the interaction in the Walecka model. Through their interaction with the nucleons these acquire the character of 'dressed' quasi-particles. This takes place in form of repeated creation and decay of virtual particle-hole and (if the Dirac sea is also taken into account) particle-antiparticle pairs which leads to a collective excitation of the whole nuclear matter system. Because a photon, emitted by an electron in a scattering process, interact with the nucleonic system via the same mechanism, these collective excitations manifest themselves as peaks in the corresponding response functions. More specific, there are four degrees of freedom: one scalar from the $\sigma$ and a longitudinal as well as two transverse from the $\omega$, where the latter are degenerated because of rotational invariance. Accordingly, two peaks are expected in the longitudinal response function (because of a mixing between the scalar and the longitudinal degree of freedom) and one in the transverse branch. These collective excitations in the time-like energy region have a direct back-coupling to the quasielastic bump in the space-like regime: they draw off strength, which causes the RPA-effect observed in former calculations [13] - 18]. In addition, these collective excitations should influence also the nucleonic properties which are studied in the heavy ion collision experiments mentioned at the beginning of this section. For example the masses of the mesons as well as the nucleons are shifted by the interaction [19] which has consequences also for the subthreshold antiproton production [20]. This shows again how deeply connected phenomena in the two different kinematic regions are.

The mechanism for the reduction of the quasielastic bump, described so far, of course makes sense only if the time-like collective excitations which draw off the strength from the space-like regime are physical degrees of freedom.

As will be shown, this is only the case if Dirac sea effects are properly taken into account. A calculation where vacuum polarization is neglected shows only one meson peak in the longitudinal and no peak at all in the transverse branch of the electro-nuclear response function. Instead we find two peaks in the upper complex energy plane which means that these are not corresponding to well defined quasi particles but announce an instability of the nuclear matter system. Therefore vacuum polarization is crucial for the stability of nuclear matter.

The purpose of the present paper is to study general properties of collective excitations in the nuclear matter system which are rather a consequence of many body effects then of the basic NN-potential actually used. Because it is not our intent to compare our curves with experimental data we use with the Walecka model a simple but nevertheless nontrivial NN-interaction. This has the advantage that for space- and time-like (real) energies the calculations may be done analytically which makes it easier to perform the numerical extension to complex energies.

In section II the criteria for the stability of collective excitations will be discussed in general. In order to explore the upper complex energy plane with respect to destabilizing unphysical modes we have to perform an analytic continuation of the electro-nuclear response functions. This will be done in section III. Using dispersion relations we introduce a renormalization concept [21] which is new in this context. Instead of using dimensional regularization - as it was done in [14] and [17] - we perform subtractions in the dispersion integrals for the polarization tensor in order to get rid of the infinities due to vacuum polarization. We show explicitly that both renormalization technics lead to the same results for the response functions which is an interesting result in itself.

In section IV the response functions are at first discussed for real energies. In section $\mathrm{V}$ we look at the single particle properties in the space-like (quasielastic bump) and the time-like regime (particle-antiparticle excitations). In both cases as a consequence of RPA-correlations we find a reduction of the response functions compared to the independent particle model. This effect is most striking for the particle-antiparticle production amplitude which for high energies is reduced exactly by a factor 2 . We show that this halving of the amplitude is a general result which holds true for any isospin independent interaction. This is one of the main results of the present paper.

In section VI we discuss the role of collective excitations extending our considerations from real energies to the 
upper complex energy plane. Neglecting vacuum polarization we find the result mentioned already above, that the nuclear matter system becomes unstable because of excitations with complex energy $z$ where $\operatorname{Im}(z)>0$. Finally our conclusions are given in section VII.

\section{STABILITY OF COLLECTIVE EXCITATIONS}

A possible mass change of light vector mesons inside nuclear matter as a consequence of medium modifications of the quark condensate $<\bar{q} q>$ [7] has recently drawn attention to calculations of timelike collective excitations in various models. An comprehensive overview of this work can be found in [9] and the references given therein. Hatsuda et. al. estimate the mass reductions of $\rho, \omega$ and $\Phi$ using QCD sum rules and compare these results with a calculation in the framework of the $\sigma \omega$-model (which is suitable extended in order to include strangeness and the $\Phi-$ meson). In the latter approach which is similar to ours in this paper they obtain the effectives masses from zeroes of the inverse meson propagator in the long wavelength limes $|\vec{q}| \rightarrow 0$ inside nuclear matter.

Apart from this actual interest in density dependent changes of the quark condensate collective modes in nuclear matter were studied already previously by several groups. The first calculation within the $\sigma \omega-$ model was performed by Chin [2]. Neglecting vacuum polarization he did an additional severe approximation 1 evaluating the momentum integrals in the expression for the dielectric function. So Chin's results are partly in contradiction to the work of Lim and Horowitz 22] who calculated the density dependent momentum integrals exactly but neglected vacuum polarization too. These authors also addressed the problem of the stability of collective modes, however from a different point of view then ours in this paper. Lim and Horowitz investigated the density dependence of real collective zero energy modes $\left(q_{0}=0\right)$ in the space-like region. If for a given density (characterized by the Fermi momentum $\left.k_{F}\right)$ and momentum $|\vec{q}|$ such a mode arises, this indicates that in the uniform nuclear matter system an instability occurs which drives a phase transition to a spatial inhomogeneous state. A similar type of instability was studied by Furnstahl and Horowitz [23] in connection with the so called Landau ghost which appears in meson propagators when vacuum polarization is included. Neither of these singularities will affect our calculations. The first type is excluded because we are only interested in nuclear matter at saturation density $\left(k_{F}=1.4 \mathrm{fm}^{-1}\right)$ where the system is stable against zero energy modes (see fig. 12 of $[22)$. The Landau ghost appears only for momentum transfers of $|\vec{q}| \geq 2.55 m(m=$ nucleon mass) 23] which is much higher then the momenta we are considering ?.

In the following we examine the nuclear matter system with respect to a different kind of instabilities. In the previous calculations of collective nuclear matter excitations mentioned above, only the denominator of the meson propagator was analyzed on the real energy axes. Zeroes of this denominator are good candidates for corresponding poles in the propagator i.e. an excitation of the system. But, while even a zero of real and imaginary parts of the denominator is not always a sufficient condition for a pole (if the nominator also becomes small), often only the vanishing of the real part for an given energy $\omega_{0}$ is already taken for an excitation. If the imaginary part of the denominator keeps sufficient small (and if the nominator is finite) this is correct and the propagator in the neighbourhood of $\omega_{0}$ has the shape of a Lorentz curve corresponding to an excitation with a decay width depending on the magnitude of the imaginary part.

In a paper by Jean et. al. 25] the denominator of the $\omega$-meson propagators has been analyzed similar to [8] and [9] in order to find a mass shift as result of medium modifications in the $\sigma \omega$-model. As it is one of the aims of the present paper, Jean et. al. also studied the influence of the Dirac sea on the effective $\omega$ mass comparing the full calculation with one where vacuum polarization is neglected and only Pauli blocking is taken into account. While in the presence of vacuum polarization all degrees of freedom are correctly reflected by a corresponding pole in the propagator they found in the latter case zeroes of the real part of the denominator simultaneously with a nonvanishing negative imaginary contribution. They concluded that "this nonzero imaginary part contributes to an unphysical (i.e. negative) decay width for the $\omega$ meson".

As mentioned above, at this point it becomes obvious that it is not sufficient to look only at the denominator of the propagator. In section VI we will show that the zero of the real part of the latter, found by Jean et. al., does not correspond to a collective excitation with "negative decay width" (whatever this means) at least not on the real energy axes. However, in order to find out what is really hidden behind this unphysical mode, it is necessary to

\footnotetext{
${ }^{1}$ As a result of this approximation, beside vacuum polarization also the density dependent Pauli blocking of NN excitations due to the filled Fermi sea is excluded from the calculation.

${ }^{2}$ In a paper by Tanaka et al. 24] it was shown that the Landau ghost is not a serious problem of relativistic many body theory because it can be eliminated in an appropriate way
} 
explore the full propagator (not only it's denominator) in the whole complex energy plane. As noticed already in the introduction, the neglect of vacuum polarization entails an instability of the nuclear matter system indicated by poles of the electro-nuclear response functions in the upper complex energy plane.

In the case of inclusive (e,e')-scattering there are two independent response functions, a longitudinal and a transverse, which can be expressed by the corresponding matrix elements of the polarization tensor $\Pi^{\mu \nu}\left(q_{0}, \vec{q}\right)$. If we choose a co-ordinate system were $\vec{q}$ is parallel to the $x$-axes, $\Pi^{\mu \nu}\left(q_{0}, \vec{q}\right)$ can be written in the form:

$$
\Pi^{\mu \nu}\left(q_{0}, \vec{q}\right)=\left(\begin{array}{cccc}
\Pi_{\mathrm{L}}\left(q_{0}, \vec{q}\right) & \frac{q_{0}}{|\vec{q}|} \Pi_{\mathrm{L}}\left(q_{0}, \vec{q}\right) & 0 & 0 \\
\frac{q_{0}}{|\vec{q}|} \Pi_{\mathrm{L}}\left(q_{0}, \vec{q}\right) & \left(\frac{q_{0}}{|\vec{q}|}\right)^{2} \Pi_{\mathrm{L}}\left(q_{0}, \vec{q}\right) & 0 & 0 \\
0 & 0 & \Pi_{\mathrm{T}}\left(q_{0}, \vec{q}\right) & 0 \\
0 & 0 & 0 & \Pi_{\mathrm{T}}\left(q_{0}, \vec{q}\right)
\end{array}\right) .
$$

The Fourier transform of $\Pi^{\mu \nu}\left(q_{0}, \vec{q}\right)$ :

$$
\Pi^{\mu \nu}(x, y)=\int_{-\infty}^{+\infty} \frac{d q_{0}}{2 \pi} \int \frac{d \vec{q}}{(2 \pi)^{3}} \Pi^{\mu \nu}\left(q_{0}, \vec{q}\right) e^{-i q_{0}\left(t_{x}-t_{y}\right)} e^{i \vec{q}(\vec{x}-\vec{y})}
$$

is defined as the change $\delta j^{\mu}(x)$ of the nuclear current in $x$ due to a change $\delta A_{\nu}(y)$ of the vector potential in $y$, induced by the scattered electron:

$$
\delta j^{\mu}(x)=\Pi^{\mu \nu}(x, y) \delta A_{\nu}(y) .
$$

According to linear response theory 26] $\Pi^{\mu \nu}(x, y)$ can be expressed by the ground state expectation value of the commutator $\left[j^{\mu}(x), j^{\nu}(y)\right]$. Causality requires that $\Pi^{\mu \nu}(x, y)$ can be non zero only if $x$ is in the forward light cone with respect to $y$, i.e. $t_{x}>t_{y}$. Including a factor (-i) which is conventional, $\Pi^{\mu \nu}(x, y)$ can be written explicitly:

$$
\Pi^{\mu \nu}(x, y)=-i<0\left|\left[j^{\mu}(x), j^{\nu}(y)\right]\right| 0>\Theta\left(t_{x}-t_{y}\right) .
$$

Here $\mid 0>$ denotes the ground state of the nuclear matter system consisting of a filled Fermi sea of nucleons above the Dirac vacuum of antiparticles.

Now, for $t_{y}>t_{x}$ the $q_{0}$-integral in (2) has to be closed in the upper complex energy plane. Then a zero contribution can only be obtained if $\Pi^{\mu \nu}\left(q_{0}, \vec{q}\right)$ is analytic there. On the contrary, if $\Pi^{\mu \nu}\left(q_{0}, \vec{q}\right)$ has poles in the upper half plane, this means that there is a response of the system preceding an external disturbance which is equivalent to an instability. Because in [2], [22], [23] only poles on the real $q_{0}$-axis were examined, non causal singularities could not be found by these authors.

\section{ANALYTIC CONTINUATION TO COMPLEX ENERGIES}

For nuclear matter (point-like Dirac particles) with an interaction mediated by a scalar and a vector meson $(\sigma \omega-$ model [1] the longitudinal and transverse electro-nuclear response functions within RPA are given by the following expressions 13 is:

$$
\begin{aligned}
& \left.\Pi_{\mathrm{RPA}}^{\mathrm{L}}\left(q_{0}, \vec{q}^{2}\right)\right)=\frac{e^{2}}{2} \Pi_{v v}^{\mathrm{L}}\left(q_{0}, \vec{q}^{2}\right) \\
& +e^{2} \frac{\left(1-\frac{q^{2}}{\vec{q}^{2}} G_{v} \Pi_{v v}^{\mathrm{L}}\right) G_{s}\left(\frac{1}{2} \Pi_{s v}\right)^{2}+\frac{1}{2} \frac{q^{2}}{\vec{q}^{2}} G_{v} G_{s} \Pi_{v v}^{\mathrm{L}} \Pi_{s v}^{2}+\frac{q^{2}}{\vec{q}^{2}}\left(1-G_{s} \Pi_{s s}\right) G_{v}\left(\frac{1}{2} \Pi_{v v}^{\mathrm{L}}\right)^{2}}{\left(1-\frac{q^{2}}{\vec{q}^{2}} G_{v} \Pi_{v v}^{\mathrm{L}}\right)\left(1-G_{s} \Pi_{s s}\right)-\frac{q^{2}}{\vec{q}^{2}} G_{v} G_{s} \Pi_{s v}^{2}}
\end{aligned}
$$

\footnotetext{
${ }^{3}$ Note that in contrast to (四) in $13 \Pi^{\mu \nu}(x, y)$ is defined as the time ordered product $-i<0\left|T j^{\mu}(x), j^{\nu}(y)\right| 0>$. For $q_{0}>0$ the Fourier transform of this expression is identical to our $\Pi^{\mu \nu}\left(q_{0}, \vec{q}\right)$ and differs only in the sign of the imaginary part for $q_{0}<0$.
} 


$$
\left[\Pi_{\mathrm{RPA}}\right]_{\mathrm{T}}\left(q_{0}, \vec{q}^{2}\right)=\frac{e^{2}}{2} \Pi_{v v}^{\mathrm{T}}\left(q_{0}, \vec{q}^{2}\right)+e^{2} \frac{G_{v}\left(q^{2}\right)\left(\frac{1}{2} \Pi_{v v}^{\mathrm{T}}\left(q_{0}, \vec{q}^{2}\right)\right)^{2}}{1-G_{v}\left(q^{2}\right) \Pi_{v v}^{\mathrm{T}}\left(q_{0}, \vec{q}^{2}\right)}
$$

with:

$$
G_{s, v}\left(q^{2}\right)=\frac{g_{s, v}^{2}}{q^{2}-m_{s, v}^{2}+i \epsilon} .
$$

$e, g_{s}$ and $g_{v}$ are the electromagnetic, scalar and vector meson coupling constant respectively. $\Pi_{a b}\left(q_{0}, \vec{q}\right)(a, b=s, v)$ is the Amplitude for a scalar or vector boson "a" to go into a nucleon-hole or nucleon-antinucleon pair which recombines to a boson " $\mathrm{b}$ " 7 . In former nuclear matter calculations $[13]-[15] \Pi_{a b}\left(q_{0}, \vec{q}\right)$ was evaluated explicitly for real $q_{0}$ employing Feynman rules. In order to get a finite vacuum contribution dimensional regularization was performed. In the following we use a method developed by Berestetskii in the context of QED [21] which is more appropriate for an analytic continuation of (5) and (6) to complex energies $z:=q_{0}+i q_{0}^{\prime}$. We start from the imaginary parts of $\Pi_{a a}$ for energy transfers $q_{0} \geq 0$. Unitarity requires that these are given by the probability of boson "a" going into a nucleon-hole or a nucleon-antinucleon pair:

$$
\begin{gathered}
\operatorname{Im} \Pi_{s s}\left(q_{0}, \vec{q}^{2}\right)=\left(4 m^{* 2}-q^{2}\right) I_{0}\left(q_{0}, \vec{q}^{2}\right)+\operatorname{Im} \Pi_{s s}^{v a c}\left(q^{2}, m^{* 2}\right) ; \\
\operatorname{Im} \Pi_{v v}^{\mathrm{L}}\left(q_{0}, \vec{q}^{2}\right)=-\vec{q}^{2} I_{0}\left(q_{0}, \vec{q}^{2}\right)+I_{2}\left(q_{0}, \vec{q}^{2}\right)+\vec{q}^{2} \operatorname{Im} \Pi_{v v}^{v a c}\left(q^{2}, m^{* 2}\right) ; \\
\operatorname{Im} \Pi_{v v}^{\mathrm{T}}\left(q_{0}, \vec{q}^{2}\right)=-\left(4 m^{* 2}+q^{2}\right) I_{0}\left(q_{0}, \vec{q}^{2}\right)-\frac{q^{2}}{\vec{q}^{2}} I_{2}\left(q_{0}, \vec{q}^{2}\right)-q_{0}^{2} \operatorname{Im} \Pi_{v v}^{v a c}\left(q^{2}, m^{* 2}\right) .
\end{gathered}
$$

The density dependent contributions to these amplitudes are given by the integrals $I_{n}$ over Fermi sea momenta up to the Fermi edge $k_{\mathrm{F}}$ :

$$
\begin{gathered}
I_{n}\left(q_{0}, \vec{q}^{2}\right)=-\frac{1}{4 \pi^{2}} \int d^{3} k \frac{n_{\vec{k}}}{\epsilon_{\vec{k}} \epsilon_{\vec{k}+\vec{q}}}\left\{\left(1-n_{\vec{k}+\vec{q}}\right)\left(2 \epsilon_{\vec{k}}+q_{0}\right)^{n} \delta\left(q_{0}-\left[\epsilon_{\vec{k}+\vec{q}}-\epsilon_{\vec{k}}\right]\right)\right. \\
\left.+\left(2 \epsilon_{\vec{k}}-q_{0}\right)^{n} \delta\left(q_{0}-\left[\epsilon_{\vec{k}+\vec{q}}+\epsilon_{\vec{k}}\right]\right)\right\} .
\end{gathered}
$$

Here $n_{\vec{k}}=\Theta\left(k_{\mathrm{F}}-|\vec{k}|\right)$ denotes the Fermi distribution of the mean field ground state and $\epsilon_{\vec{k}}=\sqrt{\vec{k}^{2}+m^{* 2}}$ is the energy of a nucleon with wave vector $\vec{k}$ and effective mass $m^{*}$. The $\delta$-functions in the integrand of (11) make sure that the two terms give a contribution only in the space-like or the time-like region respectively. The momentum integral can be easily performed analytically [13].

The Dirac sea contributions of boson "a" going into a nucleon-antinucleon pair are given by:

$$
\operatorname{Im} \Pi_{s s}^{v a c}\left(q^{2}, m^{* 2}\right)=-\frac{q^{2}}{4 \pi}\left(1-\frac{4 m^{* 2}}{q^{2}}\right)^{\frac{3}{2}} \Theta\left(q^{2}-4 m^{* 2}\right)
$$

and

$$
\operatorname{Im} \Pi_{v v}^{v a c}\left(q^{2}, m^{* 2}\right)=-\frac{1}{12 \pi}\left(1-\frac{4 m^{* 2}}{q^{2}}\right)^{\frac{1}{2}}\left(1+\frac{2 m^{* 2}}{q^{2}}\right) \Theta\left(q^{2}-4 m^{* 2}\right) .
$$

In addition to the "proper" transitions $a \rightarrow(\mathrm{ph}$ or $\mathrm{NN}) \rightarrow a(a=s, v)$ there is also a mixing of scalar and vector degrees of freedom. However, this is a purely density dependent effect. Because the vector current of the vacuum is zero, its commutator with the scalar current (which according to (何) enters the expression for the components $\Pi_{s v}$ of the polarization tensor) vanishes. The vector current of the nuclear matter system on the other side has a

\footnotetext{
${ }^{4}$ For vector bosons $\Pi_{v v} \equiv \Pi_{v v}^{\mu \nu}$ is a $4 \times 4$-tensor, the longitudinal and transverse matrix elements of which are denoted by $\Pi_{v v}^{\mathrm{L}}$ and $\Pi_{v v}^{T}$. Because we have drawn out the coupling constants and isospin factors from the vertices, the analytical form of $\Pi_{v v}$ is the same for $\omega$-mesons as for photons. A difference between $\Pi_{\omega \omega}, \Pi_{\omega \gamma}$ and $\Pi_{\gamma \gamma}$ will occur only in the numerical expressions of the vacuum contributions as a consequence of the renormalization procedure (see (20)).
} 
non-vanishing time-like component which is given by the energy density of the filled Fermi sea. The corresponding matrix element of the polarization tensor for $q_{0} \geq 0$ has the form:

$$
\operatorname{Im} \Pi_{s v}\left(q_{0}, \vec{q}^{2}\right)=2 m^{* 2} I_{1}\left(q_{0}, \vec{q}^{2}\right) .
$$

For $q_{0}<0 \operatorname{Im} \Pi_{a b}\left(q_{0}, \vec{q}^{2}\right)$ is given by the negative of the expressions (8), (9), (10) and (14) respectively:

$$
\operatorname{Im} \Pi_{a b}\left(q_{0}, \vec{q}^{2}\right)=-\operatorname{Im} \Pi_{a b}\left(\left|q_{0}\right|, \vec{q}^{2}\right) \quad\left(q_{0}<0\right),
$$

i.e. the spectral functions $\operatorname{Im} \Pi_{a b}\left(q_{0}, \vec{q}^{2}\right)$ are antisymmetric with respect to $q_{0}$.

Making use of the spectral representation:

$$
\Pi_{a b}\left(z, \vec{q}^{2}\right)=\int_{-\infty}^{\infty} \frac{d q_{0}}{\pi} \frac{\operatorname{Im} \Pi_{a b}\left(q_{0}, \vec{q}^{2}\right)}{q_{0}-z}
$$

from the analytic expressions for the imaginary parts (8)-(10) and (14) the full $\Pi_{a b}$ can be obtained in the whole complex energy plane. For $q_{0} \geq 0, \operatorname{Im} \Pi_{a b}$ can be treated as a function of $q_{0}^{2}$. Using the antisymmetry property (15) we can write (16) also in the form:

$$
\Pi_{a b}\left(z^{2}, \vec{q}^{2}\right)=\int_{0}^{\infty} \frac{d q_{0}^{2}}{\pi} \frac{\operatorname{Im} \Pi_{a b}\left(q_{0}^{2}, \vec{q}^{2}\right)}{q_{0}^{2}-z^{2}} .
$$

However (17) is directly applicable only to the density dependent portion of $\Pi_{a b}$ because this has the appropriate asymptotic behaviour for $q_{0} \rightarrow \infty$. In the high energy regime the imaginary parts of $\Pi_{s s}$ and $\Pi_{v v}$ are given by the vacuum contributions $\operatorname{Im} \Pi_{s s}^{v a c}\left(q^{2}\right)$ and $\operatorname{Im} \Pi_{v v}^{v a c}\left(q^{2}\right)$, the asymptotic behaviour of which for $q_{0} \rightarrow \infty,\left(\vec{q}^{2}=\right.$ const. $)$ follows from (12) and (13):

$$
\begin{aligned}
& \operatorname{Im} \Pi_{s s}^{v a c}\left(q_{0}^{2}, \vec{q}^{2}\right) \propto q_{0}^{2} \\
& \operatorname{Im} \Pi_{v v}^{v a c}\left(q_{0}^{2}, \vec{q}^{2}\right) \propto \text { const. }
\end{aligned}
$$

Therefore in order to get from (17) a finite result for the vacuum contributions, we have to perform in the dispersion integrals one subtraction with respect to $q_{0}^{2}$ for $\Pi_{v v}$ and two subtractions for $\Pi_{s s}$ :

$$
\begin{aligned}
\Pi_{v v}^{v a c}\left(z^{2}, \vec{q}^{2}\right)= & \Pi_{v v}^{v a c}\left(z_{r ; v}^{2}, \vec{q}^{2}\right)+\left(z^{2}-z_{r ; v}^{2}\right) \int_{0}^{\infty} \frac{d q_{0}^{2}}{\pi} \frac{\operatorname{Im} \Pi_{v v}^{v a c}\left(q_{0}^{2}, \vec{q}^{2}\right)}{\left(q_{0}^{2}-z_{r ; v}^{2}\right)\left(q_{0}^{2}-z^{2}\right)}, \\
\Pi_{s s}^{v a c}\left(z^{2}, \vec{q}^{2}\right)= & \Pi_{s s}^{v a c}\left(z_{r ; s}^{2}, \vec{q}^{2}\right)+\left.\left(z^{2}-z_{r ; s}^{2}\right) \frac{d \Pi_{s s}^{v a c}\left(z^{2}, \vec{q}^{2}\right)}{d z^{2}}\right|_{z^{2}=z_{r ; s}^{2}} \\
& +\left(z^{2}-z_{r ; s}^{2}\right)^{2} \int_{0}^{\infty} \frac{d q_{0}^{2}}{\pi} \frac{\operatorname{Im} \Pi_{s s}^{v a c}\left(q_{0}^{2}, \vec{q}^{2}\right)}{\left(q_{0}^{2}-z_{r ; s}^{2}\right)^{2}\left(q_{0}^{2}-z^{2}\right)} .
\end{aligned}
$$

Using (18) it can be easily checked that the integrals in (19) are indeed finite. This procedure is equivalent to the corresponding subtraction of infinities in the dimensional regularization method [14, 15] or any other renormalization scheme. Accordingly, the finite values of $\Pi_{v v}^{v a c}, \Pi_{s s}^{v a c}$ and $d \Pi_{s s}^{v a c} / d z^{2}$ have to be fixed at appropriate renormalization points $z_{r}^{2}$. We have chosen to follow the "on mass shell" renormalization of [14]

\footnotetext{
${ }^{5}$ There is a certain arbitrariness in the renormalization procedure for effective field theories because it can be argued that the masses of the scalar and vector bosons, mediating the interaction in these theories, do not have to be identical with the masses of physical mesons. Thus some authors 15], 23. prefer to take e.g. $q^{2}=0$ as renormalization point . Fortunately the numerical results for the response functions are only slightly changed by this different choice.
} 


$$
\begin{aligned}
\Pi_{\gamma \gamma}^{v a c}\left(z_{r ; \gamma}^{2}=\vec{q}^{2}, m^{*}=m\right) & =0 \\
\Pi_{\omega \gamma}^{v a c}\left(z_{r ; \omega}^{2}=\vec{q}^{2}+m_{\omega}^{2}, m^{*}=m\right) & =0 \\
\Pi_{\omega \omega}^{v a c}\left(z_{r ; \omega}^{2}=\vec{q}^{2}+m_{\omega}^{2}, m^{*}=m\right) & =-\left.m_{\omega}^{2} \frac{d \Pi_{\omega \omega}^{v a c}\left(z^{2}, m^{*}=m\right)}{d z^{2}}\right|_{z_{r ; \omega}^{2}=\vec{q}^{2}+m_{\omega}^{2}} \equiv \delta m_{\omega}^{2} \\
\Pi_{\sigma \sigma}^{v a c}\left(z_{r ; \sigma}^{2}=\vec{q}^{2}+m_{\sigma}^{2}, m^{*}=m\right) & =\left.\frac{d \Pi_{\sigma \sigma}^{v a c}\left(z^{2}, m^{*}=m\right)}{d z^{2}}\right|_{z_{r ; \sigma}^{2}=\vec{q}^{2}+m_{\sigma}^{2}}=0 .
\end{aligned}
$$

Here $\delta m_{\omega}^{2}$ denotes a mass shift term which is introduced in order to keep the pole of the renormalized $\omega$-propagator in the vacuum at the known mass of $m_{\omega}=783 \mathrm{MeV}$. With the boundary conditions (20) the (finite) vacuum contributions to the response functions (19) are completely determined in the whole complex energy plane.

\section{RESPONSE FUNCTIONS}

Having obtained explicit expressions for $\Pi_{a b}\left(z^{2}, \vec{q}^{2}\right)$, the analytic continuation of the RPA-expressions (5) and (6) can be easily obtained by the substitution $q_{0} \rightarrow z=q_{0}+i q_{0}^{\prime}$. On the contrary, the original expressions (5) and (6) can be recovered from $\Pi_{\mathrm{RPA}}\left(z, \vec{q}^{2}\right)$ 同 by the prescription:

$$
\Pi_{\mathrm{RPA}}\left(q_{0}, \vec{q}^{2}\right)=\Pi_{\mathrm{RPA}}\left(z=q_{0}+i \epsilon, \vec{q}^{2}\right) .
$$

Here it should be noted that the extrapolation to complex energies (17) is even necessary to obtain the correct result for the response function on the real energy axis. The reason is, that the $\delta$-like peaks in $I_{m} \Pi_{\mathrm{RPA}}$ which are caused by the collective excitations may be calculated only taking the proper limes according to (19) (see section VI).

The numerical values of the parameters of the theory are given in [1] :

$$
\begin{array}{cc}
m_{s}=550 \mathrm{MeV} & m_{v}=783 \mathrm{MeV} \\
g_{s}^{2}=\left\{\begin{array}{lll}
92.87 & \text { (MFA) } \\
69.87 & (\mathrm{HA})
\end{array} g_{v}^{2}= \begin{cases}135.7 & (\mathrm{MFA}) \\
79.8 & (\mathrm{HA})\end{cases} \right.
\end{array}
$$

The coupling constants are adjusted to the saturation property of nuclear matter at an energy of $-16 \mathrm{MEV}$ per nucleon for a density corresponding to a Fermi momentum of $k_{\mathrm{F}}=280 \mathrm{MEV} / c$. While in mean field approximation (MFA) vacuum effects are neglected, the Hartree approximation includes these properly. The corresponding values for the effective mass of the nucleon are given by $m^{*}=0.56 m$ (MFA) and $m^{*}=0.78 m$ (HA) respectively.

Performing the limiting procedure according to (21) the electro-nuclear response functions can be obtained from $\Pi\left(z, \vec{q}^{2}\right)$ :

$$
\mathrm{R}_{\mathrm{L}, \mathrm{T}}\left(q_{0}, \vec{q}^{2}\right)=-\frac{V}{\pi} \operatorname{Im} \Pi_{\mathrm{L}, \mathrm{T}}\left(q_{0}+i \epsilon, \vec{q}^{2}\right) .
$$

\section{SINGLE PARTICLE PROPERTIES FOR SPACE- AND TIMELIKE MOMENTUM TRANSFERS}

Part a) of figs. 1-4 show $R_{\mathrm{L}}$ and $\mathrm{R}_{\mathrm{T}}$ for a momentum transfer of $|\vec{q}|=1.5 k_{\mathrm{F}}$ which was a typical value in former electron scattering experiments [10], [11]. Part b) of the figures show the corresponding real portions $\operatorname{Re} \Pi_{\mathrm{L}}$ and $\operatorname{Re} \Pi_{\mathrm{T}}$ of the longitudinal and transverse matrix elements of the polarization tensor (see equ. (11)). The energy ranges from the space-like region $\left(q_{0}<|\vec{q}|\right)$ up to the deep time-like regime $\left(q_{0}>|\vec{q}|\right)$. While vacuum polarization is included in figs. 3 and 4, in figs. 1 and 2 purely density dependent contributions are shown. As mentioned already in section I, our goal is to point out qualitative differences between these two approximations rather then to compare the results with experimental data (which for the quasielastic regime - where such data are only available up to now - has been done by several authors [16], [17], [18, [23]). Therefore we did not include form factors but performed the calculations for

\footnotetext{
${ }^{6}$ The same holds true of course for $\Pi_{a b}$ according to (19)
} 
point-like Fermions in nuclear matter. The dashed curves show the results including only one polarization bubble (corresponding to the first term in equs. (5) and (6) respectively), while in the full curves RPA-correlations are taken into account according to the the second expressions in (5) and (6). The structure on the left sides $\left(\omega / k_{\mathrm{F}} \lesssim 1\right)$ of the four figures represents the quasielastic bump which is measured in (e,e') scattering experiments. According to (22) the effective mass $m^{*}$ is higher when vacuum contributions are included. This causes the quasielastic peak to be a bit narrower in this case [14, 15]. The cusp at $\omega / k_{\mathrm{F}} \sim 0.18$ in the RPA-curve of fig. 1a is due to an almost zero point of the denominator in equ. (5) which indicates that long-range correlations are important.

From figs. 1a and 2a one easily reads off that the purely density dependent contributions to the response functions are zero for $\omega \rightarrow \infty$. Therefore Re $\Pi_{\mathrm{L}, \mathrm{T}}$ in figs. $1 \mathrm{~b}$ and $2 \mathrm{~b}$ can be obtained from the curves in Figs. 1a and 2a directly (i.e. without any subtractions) by means of equs. (17) and (23). The quasielastic bump in the response functions has its counterpart in a 'oscillator-structure' in the real parts of $\Pi_{\mathrm{L}, \mathrm{T}}$. The cusp at $\omega / k_{\mathrm{F}} \sim 0.18$ in the RPA-curve of fig. 1a corresponds to a small 'shoulder' at the same energy in fig. $1 \mathrm{~b}$.

With vacuum polarization as well as without, many body effects which are taken into consideration in the RPA provide a considerable reduction of the longitudinal and a smaller decrease of the transverse response functions. The reason for this suppression in the space-like region has its origin in the time-like regime and consequently keeps hidden for electron scattering experiments: strength is transfered to collective modes which in the RPA-curves appear as sharp peaks in the so called 'unphysical region' $|\vec{q}|<\omega<\sqrt{4 m^{* 2}+\vec{q}^{2}}$. These peaks will be discussed in more detail below. Despite the RPA-curves are quite different in shape, the omission of vacuum polarization does not change drastically the qualitative picture in the quasi-free regime. Comparing figs. 1 and 2 with figs. 3 and 4 one sees that the last statement holds true for the response functions itself as well as for the the real parts of $\Pi_{\mathrm{L}, \mathrm{T}}$.

This changes completely in the time-like energy range. For $q_{0} \geq \sqrt{4 m^{* 2}+\vec{q}^{2}}$ the production of NN- pairs from the Dirac sea becomes possible. According to (9) and (13) for the longitudinal response (fig. 3a) this vacuum polarization has a "square root-like" $q_{0}$-dependence (but the slope keeps finite) at threshold and approaches a constant value for increasing energies. Đ

From equs. (9) and (10) one finds for $q_{0}>>|\vec{q}|$ as a relation between the longitudinal and transverse matrix elements of $\operatorname{Im} \Pi_{v v}^{\mu \nu}$ :

$$
\frac{\operatorname{Im} \Pi_{v v}^{\mathrm{T}}\left(q_{0}^{2}, \vec{q}^{2}\right)}{\operatorname{Im} \Pi_{v v}^{\mathrm{L}}\left(q_{0}^{2}, \vec{q}^{2}\right)} \sim \frac{q_{0}^{2}}{\vec{q}^{2}} \sim \frac{q^{2}}{\vec{q}^{2}}
$$

As will be shown below, for high energies not only the one bubble contribution but also the RPA-corrections to $\Pi_{\mathrm{RPA}}^{\mathrm{L}, \mathrm{T}}$ in (5) and (6) are proportional to $\Pi_{v v}^{\mathrm{L}, \mathrm{T}}$ and consequently also obey the relation (24) $\mathrm{P}$. Hence vacuum contribution to $\mathrm{R}_{\mathrm{T}}$ in fig. 4a in comparison with $\mathrm{R}_{\mathrm{L}}$ in fig. 3a is weighted by an additional factor $q^{2}$ which causes a rapid increase for energies above threshold. So in order to show the low and high energy branches in the same figure we had to scale the transverse $\mathrm{N} \overline{\mathrm{N}}$-contribution by a factor 0.05 .

In the presence of nuclear matter the production of particle-hole pairs from the Dirac sea is inhibited if the final state has momentum lower then $k_{\mathrm{F}}$. So the Fermi sea causes a reduction of the vacuum amplitude for the process $e^{+} e^{-} \rightarrow \mathrm{N} \overline{\mathrm{N}}$. Figs. 1a and 2a show this negative density dependent contribution to the longitudinal and transverse response function in the time-like energy regime. The big slope of $\mathrm{R}_{\mathrm{L}, \mathrm{T}}$ at threshold is reflected as a cusp structure in the real parts of $\Pi_{\mathrm{L}, \mathrm{T}}$ (see figs. $1 \mathrm{~b}$ and $2 \mathrm{~b}$ ). Again the transverse branch is weighted by an additional factor $q^{2}$ (compared to the longitudinal contribution: see equs. (9) and (100) which results in an enhancement of the corresponding time-like counterpart of the quasielastic peak.

From figs. 1a and 2a it is easily read off that approximations which neglect vacuum polarization suffer from the deficiency that the response function is no more positive definite over the whole energy range. The sum of vacuum and density dependent contributions (figs. 3a and 4a) however is always positive P. As noticed already in sec. III, with

\footnotetext{
${ }^{7}$ Note that the curves in figs. $3 \mathrm{a}$ and $4 \mathrm{a}$ show the sum of vacuum polarization and the corresponding density dependent contribution. But it follows from (8)-(11) (and can be explicitly read off from figs. 1a and 2a) that in the time-like region the latter are nonzero only for the small energy range $\min \left(2 \epsilon_{|\vec{q}| / 2}, \epsilon_{\vec{k}_{\mathrm{F}}-|\vec{q}|}+\epsilon_{k_{\mathrm{F}}}\right) \leq q_{0} \leq \epsilon_{\vec{k}_{\mathrm{F}}+|\vec{q}|}+\epsilon_{k_{\mathrm{F}}}$. So for higher energies in the presence of nuclear matter the shape of the curves is the same as for the vacuum.

${ }^{8}$ From the solid and dashed curves in figs. 3 and 4 one sees already that in the high energy limes the two approximations can differ only by a multiplicative factor which - as will be proven analytically below - has the value $1 / 2$. Therefore in the following considerations on the asymptotic behaviour of the polarization propagator we do not have to distinguish between $\Pi_{\mathrm{RPA}}^{\mathrm{L}, \mathrm{T}}$ and $\Pi_{v v}^{\mathrm{L}, \mathrm{T}}$.

${ }^{9}$ If one compares figs. 1a and 2a with figs. $3 \mathrm{a}$ and $4 \mathrm{a}$ it seems that the (negative) time-like density dependent contributions
} 
regard to vacuum polarization $\Pi_{v v}^{\mathrm{L}}$ does not fall off to zero for $q_{0} \rightarrow \infty$ but approaches a constant value (see equ. (18)). As a consequence of our remarks above this holds true also for the longitudinal response function (see fig. 3a). Therefore the real part of $\Pi_{\mathrm{L}}$ in fig. 3b cannot be obtained directly from the curve in fig. 3a making use of (17) as it was the case in fig 1 . Instead, according to the first equation of (19) one subtraction has to be performed. The meaning of this renormalization procedure becomes more obvious, if one splits up the vacuum contribution in fig. 3a in a constant part (which starts at the $\mathrm{N} \overline{\mathrm{N}}$-threshold) and a contribution which has an asymptotic behaviour like $1 / q_{0}^{2}$ for $q_{0} \rightarrow \infty$ (compare equ. (13) ). More specifically we write $\mathrm{R}_{\mathrm{L}}\left(q_{0}^{2}, \vec{q}^{2}\right)$ in the form:

$$
\mathrm{R}_{\mathrm{L}}\left(q_{0}^{2}, \vec{q}^{2}\right)=\mathrm{R}_{\mathrm{L}}\left(q_{0}^{2}=\infty, \vec{q}^{2}\right) \Theta\left(q^{2}-4 m^{* 2}\right)+\Delta \mathrm{R}_{\mathrm{L}}\left(q_{0}^{2}, \vec{q}^{2}\right)
$$

where by construction $\Delta \mathrm{R}_{\mathrm{L}}\left(q_{0}^{2}, \vec{q}^{2}\right)$ provides a finite contribution to the integral (17) which is harmless. The leading order high energy contribution to $R e \Pi_{L}$ comes from the $\Theta$-function which may be integrated performing one subtraction:

$$
\operatorname{Re} \Pi_{\mathrm{L}}\left(q_{0}^{2}, \vec{q}^{2}\right) \sim-q_{0}^{2} P \int_{4 m^{*}+\vec{q}^{2}}^{\infty} \frac{d q_{0}^{\prime 2}}{\pi} \frac{1}{q_{0}^{\prime 2}\left(q_{0}^{\prime 2}-q_{0}^{2}\right)} \sim \ln \left(q_{0}^{2}\right) .
$$

The negative sign of the principal value integral in (26) follows from the definition (23) of the response function: $\mathrm{R}_{\mathrm{L}} \sim-\operatorname{Im} \Pi_{\mathrm{L}}>0$. In this way we obtain the same result as by using dimensional regularization 14], 17].

Because of (24) two subtractions are needed in order to calculate $\operatorname{Re} \Pi_{\mathrm{T}}\left(q_{0}^{2}, \vec{q}^{2}\right)$ from the response function in fig. 4a (i.e. one has to integrate $\mathrm{R}_{\mathrm{T}}\left(q_{0}^{2}, \vec{q}^{2}\right) / q_{0}^{4}$ and multiply the result with $\left.q_{0}^{4}\right)$. Analogous to (26) one obtains to leading order in $q_{0}^{2}$ :

$$
\operatorname{Re} \Pi_{\mathrm{T}}\left(q_{0}^{2}, \vec{q}^{2}\right) \sim q_{0}^{2} \ln \left(q_{0}^{2}\right) .
$$

This result can be easily checked in fig. 4b. Hence the relation (24) is valid also for the real parts of $\Pi_{\mathrm{L}, \mathrm{T}}$, i.e. in the high energy limit we get the final relation:

$$
\Pi_{\mathrm{T}}\left(q_{0}^{2}, \vec{q}^{2}\right) \sim q^{2} \Pi_{\mathrm{L}}\left(q_{0}^{2}, \vec{q}^{2}\right)
$$

which will be used in the following 10 .

RPA-correlations lead to a dramatical decrease of $N \bar{N}$ pair production by a factor $1 / 2$. In our approximation of point-like nucleons without anomalous magnetic moment this suppression is a consequence of isospin symmetry. For the transverse response function this can be read off most easily from equ. (6). Because the photon in our approximation couples only to protons while meson exchange is charge-independent, the 'electromagnetic bubbles' $\Pi_{v v}^{\mathrm{T}}$ for single particle-hole pair creation as well as in the numerator of the RPA-correction are weighted by a factor $1 / 2$ in contrast to the 'mesonic' bubble in the denominator. For high energies the $q^{2}$-dependent part of this denominator becomes much larger than 1 . This can be checked substituting $\Pi_{v v}^{\mathrm{T}}$ according to (28): the leading factor $q^{2}$ cancels the $1 / q^{2}$-decrease of the propagator $G_{v}\left(q^{2}\right)$, so that the RPA-denominator in (6) goes like $\left(1-g_{v}^{2} \Pi_{v v}^{\mathrm{L}}\right) \sim 1-g_{v}^{2} \ln \left(q_{0}^{2}\right)$ (see equ. (26)). Hence for high energies the 1 in the RPA-denominator may be neglected. Then the rest of the denominator in (6) cancels against the corresponding part in the nominator so that (because of the isospin-factor $1 / 2)$ one gets from the RPA-correction a contribution which is exactly $(-1 / 2)$ times the single NN -bubble contribution П. The same holds true for the longitudinal part (5) of the polarization propagator. As pointed out in the section preceding equ. (14), $\Pi_{s v}$ is a purely density dependent contribution. From equ. (11) it follows (and can be easily checked in figs. 1 and 2) that $\Pi_{s v}\left(q_{0}, \vec{q}^{2}\right)=0$ for $q_{0}>\epsilon_{\vec{k}_{\mathrm{F}}+|\vec{q}|}+\epsilon_{k_{\mathrm{F}}}$. Consequently only the third term in the nominator and the first term in the denominator of (5) are nonzero for $q_{0}$ high enough. So in this limes $\Pi_{\mathrm{RPA}}^{\mathrm{L}}$ can be written:

start at $q_{0} \sim 4 k_{\mathrm{F}}$ which is about $1 k_{\mathrm{F}}$ below the threshold for pair production. But one has to keep in mind, that these curves show the result of two different approximations. As was pointed out in section IV the effective nucleon mass is higher when vacuum polarization is taken into account properly. Consequently the NN-threshold $\min \left(2 \epsilon_{|\vec{q}| / 2}, \epsilon_{\vec{k}_{\mathrm{F}}-|\vec{q}|}+\epsilon_{k_{\mathrm{F}}}\right)$ is $\operatorname{shifted}$ according to the corresponding mass shift from $m^{*}=0.56 m$ to $m^{*}=0.78 m$. So in figs. 3 a and 4 a the density dependent contributions start at the same threshold as vacuum polarization

${ }^{10}$ Here of course we recover the tensor structure: $\Pi_{v a c}^{\mu \nu}=\left[g^{\mu \nu}-q^{\mu} q^{\nu} / q^{2}\right] \Pi_{v a c}\left(q^{2}\right)$. So equ. (2\&) proves that our renormalization procedure preserves current conservation.

${ }^{11}$ Note that the starting energy of this asymptotic behaviour depends on the numerical value of $g_{v}^{2}$ which is a parameter of the model. If $g_{v}^{2}>>1$ (compare equ. (22)) the reduction by a factor $1 / 2$ starts practically already at threshold which is confirmed by figs. 3 and 4 . 


$$
\left.\left[\Pi_{\mathrm{RPA}}\right]_{\mathrm{L}}\left(q_{0}, \vec{q}^{2}\right)\right)=\frac{e^{2}}{2} \Pi_{v v}^{\mathrm{L}}\left(q_{0}, \vec{q}^{2}\right)+e^{2} \frac{\frac{q^{2}}{\bar{q}^{2}} G_{v}\left(\frac{1}{2} \Pi_{v v}^{\mathrm{L}}\right)^{2}}{\left(1-\frac{q^{2}}{\vec{q}^{2}} G_{v} \Pi_{v v}^{\mathrm{L}}\right)} .
$$

Completely analogous to the RPA-correction to $\Pi_{\mathrm{T}}$, the 1 in the denominator of $(29)$ may be neglected and one gets a negative contribution of just one half of the single pair production amplitude.

This argumentation on the one hand is very general because it is based only on the isospin symmetry of the NNinteraction and holds true beyond the $\sigma \omega$-model. So for point-like nucleons we would expect a suppression of the cross section $e^{+} e^{-} \rightarrow$ p $\bar{p}$ by $50 \%$, inside nuclear matter as well as in the vacuum. On the other hand we know that nucleons are not point-like particles, which may invalidate (at least partly) our reasoning. Nevertheless, we think that our model calculation has shown that many body correlations can produce effects far beyond the usually assumed level of 10-20\%. This result should not alter dramatically taking the finite size of nucleons properly into account.

An observable which would be directly affected by such many body effects is the ratio:

$$
\mathrm{R}=\frac{\sigma\left(e^{+} e^{-} \rightarrow \text { hadrons }\right)}{\sigma\left(e^{+} e^{-} \rightarrow \mu^{+} \mu^{-}\right)}
$$

Within the simple quark-parton model (QPM) - which corresponds to the one bubble approximation in our calculations $-\mathrm{R}$ is given by:

$$
\mathrm{R}=3 \sum_{q} e_{q}^{2}
$$

where the index $q$ runs over all quark flavours with charge $e_{q}$. A review on the value of $\mathrm{R}$ measured in several experiments can be found in [27]. Unfortunately our considerations concerning the process $e^{+} e^{-} \rightarrow$ p $\bar{p}$ cover only a fraction of $\mathrm{R}$ which makes it difficult to compare directly with these experiments. Nevertheless, a thorough analysis of the above general isospin argument on the quark level seems to be worthwhile but goes beyond the scope of the present paper.

\section{COLLECTIVE PHENOMENA}

Another remarkable difference between the full and dashed curves in figs. 1-4 are the narrow peaks in the time-like energy regime below the threshold for pair production. These belong to the collective excitations already discussed in sections I and II. The correct structure of these poles in the real and imaginary part of the polarization propagator may be obtained only if the calculation is extended to complex energies. The sharp $\delta$-peaks in the response functions (see part a of figs. 1-4) are the result of an analytic continuation back to the real energy axis according to (23). For $\operatorname{Im}(z)=0$ the 'one bubble' contribution to the response function is zero in the unphysical region $|\vec{q}| \leq q_{0} \leq$ $\min \left(2 \epsilon_{|\vec{q}| / 2}, \epsilon_{\vec{k}_{\mathrm{F}}-|\vec{q}|}+\epsilon_{k_{\mathrm{F}}}\right)$ 巴2. As a consequence, also the nominator of the RPA-corrections in equs. (5) and (6) vanishes for these energies. Hence zeros of the denominator, which are the signal of collective excitations, lead to an indefinite expression for the RPA-formulas.

Former calculations of the electronuclear response functions [13]- [18] were restricted only to real spacelike energies (more specific: to the quasi elastic bump) and therefore did not have to care about this problem. On the other hand, the authors who looked for timelike excitations [2], [9], [22], 225] only calculated the denominators of equs. (5) and (6) and looked for zeroes in this quantity. As will be shown below, for real excitations this method provides the correct spectrum, but in the case of an instability such a treatment may be misleading.

From counting of degrees of freedom (see section I) one would expect two peaks in the longitudinal and one (twofold degenerate) peak in the transverse response function. But figs. 1 and 2, where only density dependent contributions are taken into account, show one single excitation in the longitudinal and none at all in the transverse branch. However, including vacuum polarization the curves in figs. 3 and 4 show the correct number of peaks.

Here it is instructive to look at the RPA-denominators in both approximations which are shown in fig. 5 for the longitudinal branch (the following arguments hold true also for the transverse response). The large peaks in the real parts of both figures come from the free $\sigma$ and $\omega$ propagator in the denominator of equ. (5) with masses given in equ. (22). The dispersion of the dressed mesons inside nuclear matter is determined by the zeroes of the full curves in

\footnotetext{
${ }^{12}$ There are no single particle excitations in this energy range
} 
fig. 5 (but, as said before, only if the imaginary part, i.e. the dashed curve in fig. 5 , vanishes too). Including vacuum polarization (fig. $5 \mathrm{~b}$ ) we find a reduction of the effective meson masses because the zeroes are situated below the peaks of the free propagators. This result is in accordance with previous calculations [8], [9], 25]. On the contrary, neglecting Dirac sea effects, the real zeroes of the RPA-denominator (from which only the first corresponds to a well defined excitation) are situated above the free meson poles which means an enhanced mass of the quasiparticles.

In order to understand the reason for this difference in the two approximations it is helpful to take a closer look at the shape of the peaks in fig. 5 . If one compares fig. $5 \mathrm{a}$ with fig. $5 \mathrm{~b}$ it is easy to verify a different sign of the meson poles. While in fig. $5 \mathrm{a}$ the real part of the denominator is positive below the poles and negative above, the zeroes corresponding to the quasiparticles also lie above the poles. Exactly opposite is the situation for the calculation including vacuum polarization (fig. 5b) which leads to the reduction of the meson mass.

Now, the mechanism how Dirac sea effects cause this negative mass shift becomes clearer, if one looks at the denominator of the RPA correction in equ. (5). In the energy range between the photon point $q^{2}=0$ and the threshold for pair production $q^{2}=m^{* 2}$ the mixing term of scalar and vector degrees of freedom $\Pi_{s v}^{2}$ can be neglected in comparison with the pure terms $\Pi_{v v}^{\mathrm{L}}$ and $\Pi_{s s}$. So for these energies the RPA denominator may be approximated by the expression $\left(1-\frac{q^{2}}{\vec{q}^{2}} G_{v} \Pi_{v v}^{\mathrm{L}}\right)\left(1-G_{s} \Pi_{s s}\right)$. The poles of the propagators $G_{s}$ and $G_{v}$ provide the peaks discussed above, the signs of which depend on the sign of $\Pi_{v v}^{\mathrm{L}}$ and $\Pi_{s s}$ in the neighbourhood of these poles respectively. Because without RPA corrections $\Pi_{v v}^{\mathrm{L}}$ is directly proportional to the longitudinal response function (see the first term in equ. (5)) its behaviour can be red off from the dashed curves in figs. 1 and 3. For the energies under consideration the imaginary parts vanish. Neglecting the Dirac sea, the dashed curve in fig. $1 \mathrm{~b}$ for $\vec{q}^{2}<q_{0}^{2}<\vec{q}^{2}+m^{* 2}$ has positive sign. Because the scalar and vector meson masses are well separated, near the vector pole $\left(1-G_{s} \Pi_{s s}\right) \sim 1>0$. This leads to the peak structure described above and an enhancement of the $\omega$ mass inside nuclear matter in this approximation.

Comparing fig. $1 \mathrm{~b}$ with fig. $3 \mathrm{~b}$ it is easy to see, that vacuum polarization provides a negative contribution to the real part of $\Pi_{v v}^{\mathrm{L}}$. The result of this is the switch of sign in the free $\omega$ pole observed in fig. $5 \mathrm{~b}$ with the consequence of a reduced mass of the dressed quasiparticle. According to equ. (19) the magnitude of the negative Dirac sea contribution and thus also the meson mass shift depends on the amplitude for nucleon-antinucleon pair production. The threshold for this process inside nuclear matter is determined by the effective nucleon mass $m^{*}$ which is the free nucleon mass $m$ reduced by a term proportional to the scalar density $\langle\bar{\psi} \psi>[\mathbb{1}$. Hence analogous to the results of the QCD inspired approach [7], 9] with a meson mass shift due to medium modifications of the quark condensate $<\bar{u} u>$ also in the Walecaka model a scalar density is responsible for the changes in the dispersion of the mesonic degrees of freedom.

Despite the preceding considerations, as an illustration of our method, gave us a qualitative picture of the underlying mechanism for the meson mass shift, it is not the purpose of the present paper to analyze this effect in more detail. Rather it is our goal to point out the importance of vacuum polarization for the stability of the nuclear matter system. In [25] Jean et. al. argue that, neglecting vacuum polarization, the zero in the real part of fig. $5 \mathrm{a}$ at $\omega / k_{\mathrm{F}} \sim 4.5$ belongs to a collective excitation with "an unphysical (i.e. negative) decay width" because of the nonzero negative imaginary part of the RPA-denominator in this energy range. However, according to figs. 1 and 2 for real energies the response functions itself do not show any sign of such a unphysical mode. The cusp like structures at $\omega / k_{\mathrm{F}} \sim 4$ in the real parts of the longitudinal and transverse matrix elements of the polarization tensor (figs. $1 \mathrm{~b}$ and $2 \mathrm{~b}$ ) are already present in the one bubble approximation and therefore are not the result of collective phenomena.

The true situation unfolds itself only if the calculation of the response functions is extended to complex energies (see figs. 6 and 7). The peaks which are missed on the real energy axes reappear as undamped poles in the upper complex half plane. So in contrast to the prediction of [25] an approximation which neglects vacuum polarization does not lead to a negative decay width of the $\omega$ meson but to collective excitations which are unphysical because, according to the discussion in section II, they manifestly destroy causality. Consequently calculation within this approximation cannot provide reliable results.

\section{CONCLUSIONS}

We studied qualitative properties of the electro-nuclear response functions for nuclear matter with respect to single particle and collective excitations. Performing the calculations exemplary in Walecka's $\sigma \omega$-model, the main results were obtained from general arguments which should hold true far beyond this simple model for the medium NN-interaction.

In contrast to former calculations where the main attention was concentrated on the quasielastic region, we extended our considerations 1) to the time-like energy regime and 2) to complex energies. The latter was achieved making use of dispersion relations. As an alternative to dimensional regularization we introduced a renormalization scheme which is more appropriate to our approach: infinite vacuum contributions were removed performing suitable subtractions 
in the dispersion integrals. With the appropriate boundary conditions this method leads to the same results as dimensional regularization.

Neglecting vacuum polarization we found that there is a violation of causality because of unstable longitudinal and transverse collective excitations in the upper complex energy plane. Including vacuum polarization, these collective modes are shifted to the real axes and change to proper excitations of the system. So we have shown that vacuum polarization is crucial for the stability of nuclear matter.

In addition we have shown that RPA-corrections reduce the production amplitude of $p \bar{p}$ pairs by a factor $1 / 2$. This result is remarkable because it holds true for any interaction between point-like nucleons which preserves isospin symmetry. Our argument may have interesting consequences for the process $e^{+} e^{-} \rightarrow \mathrm{p} \overline{\mathrm{p}}$.

[1] B.D. Serot and J. D. Walecka, Adv. Nucl. Phys., Vol. 16, eds. J. W. Negele and E. Vogt (Plenum, New York, 1986).

[2] S.A. Chin, Ann. Phys. (N.Y.) 108, 301 (1977).

[3] A. Schroeter et al. Nucl. Phys. A553, 775c (1993).

[4] D. Beavis et al. Phys. Rev. Lett. 753633 (1995).

[5] T. Alber et al. Phys. Lett. B 366, 56 (1996).

[6] For an overview see the articles in Proceedings of Quark Matter '93 [Nucl. Phys. A566 (1994)], Quark Matter '95 [Nucl. Phys. A590 (1995)] and Quark Matter '96 [Nucl. Phys. A610 (1996)]

[7] G. Q. Li, C. M. Ko, G.E. Brown Nucl. Phys. A606 (1996)

[8] H. Shiomi and T. Hatsuda, Phys. Lett. B 334, 281 (1994).

[9] T. Hatsuda, H. Shiomi and H. Kuwabara, Prgr. Theor. Phys. 951009 (1996).

[10] Z. E. Meziani et al. Phys. Rev. Lett. 522130 (1984).

[11] C. C. Blatchley et al. Phys. Rev. C 441243 (1986).

[12] J. P. Chen et al. Phys. Rev. Lett. 661283 (1991).

[13] H. Kurasawa and T. Suzuki, Nucl. Phys. A 445685 (1985).

[14] H. Kurasawa and T. Suzuki, Nucl. Phys. A 490571 (1988).

[15] C. J. Horowitz, Phys. Lett. B 208, 8 (1988).

[16] K. Wehrberger and F. Beck, Phys. Rev. C 35, 2337 (1987).

[17] K. Wehrberger and F. Beck, Nucl. Phys. A 4912337 (1987).

[18] C. J. Horowitz and J. Piekarewicz, Nucl. Phys A 511, 461 (1990).

[19] K. Saito and A.W. Thomas Phys. Rev. C 51, 2757 (1995).

[20] G. Q. Li and C. M. Ko Phys. Rev. C 50, 1725 (1994).

[21] V. B. Berestetskii, E. M. Lifshitz and L. P. Pitaevskii Quantum Electrodynamics (Pergamon Press, Oxford 1982).

[22] K. Lim and C. J. Horowitz, Nucl. Phys A 501, 729 (1989).

[23] R. J. Furnstahl and C. J. Horowitz, Nucl. Phys A 485, 632 (1988).

[24] K. Tanaka, W. Bentz, A. Arima and F. Beck, Nucl. Phys A 528, 676 (1991).

[25] H.-C. Jean, J. Piekarewicz and A. G. Williams Phys. Rev. C 49, 1981 (1994).

[26] see e.g.: A. L. Fetter and J. D. Walecka, Quantum Theory of Many-Particle Systems (Mc Graw-Hill, New York 1971).

[27] R. Marshall, in: High Energy Electron-Positron Physics, eds. A. Ali and P.Soeding (Singapore: World Scientific 1988), 141. 

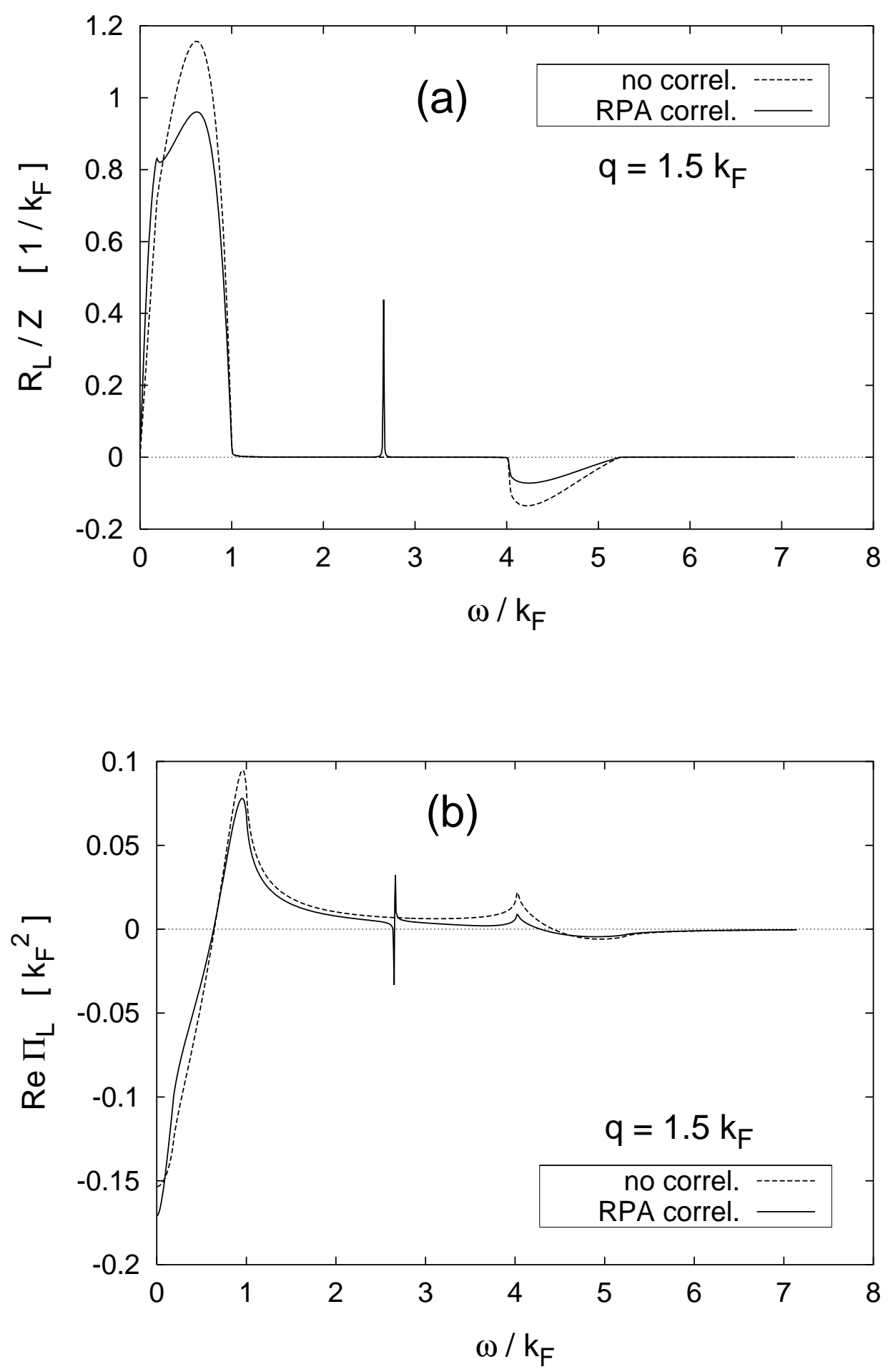

FIG. 1. (a) Longitudinal response function and (b) real part of the longitudinal matrix element $\Pi_{\mathrm{L}}$ of the polarization tensor for nuclear matter at a momentum transfer of $1.5 k_{\mathrm{F}}$ without vacuum polarization. The dashed curve shows a calculation for space-like and time-like energies neglecting RPA-correlations, while these are included in the full curve. 

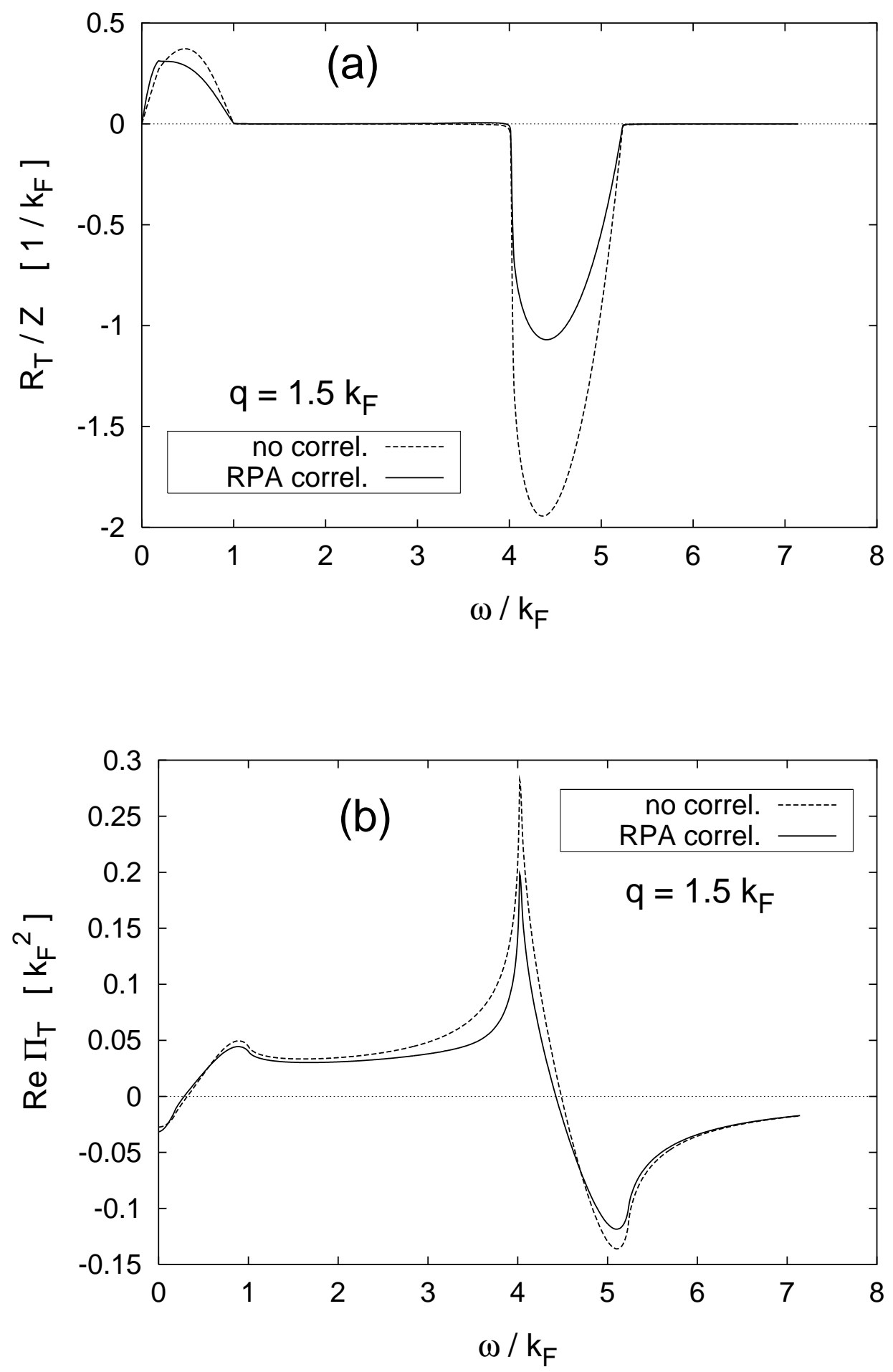

FIG. 2. Same as fig. 1 for (a) the transverse response function and (b) the real part of the transverse matrix element $\Pi_{\mathrm{T}}$ of the polarization tensor. 

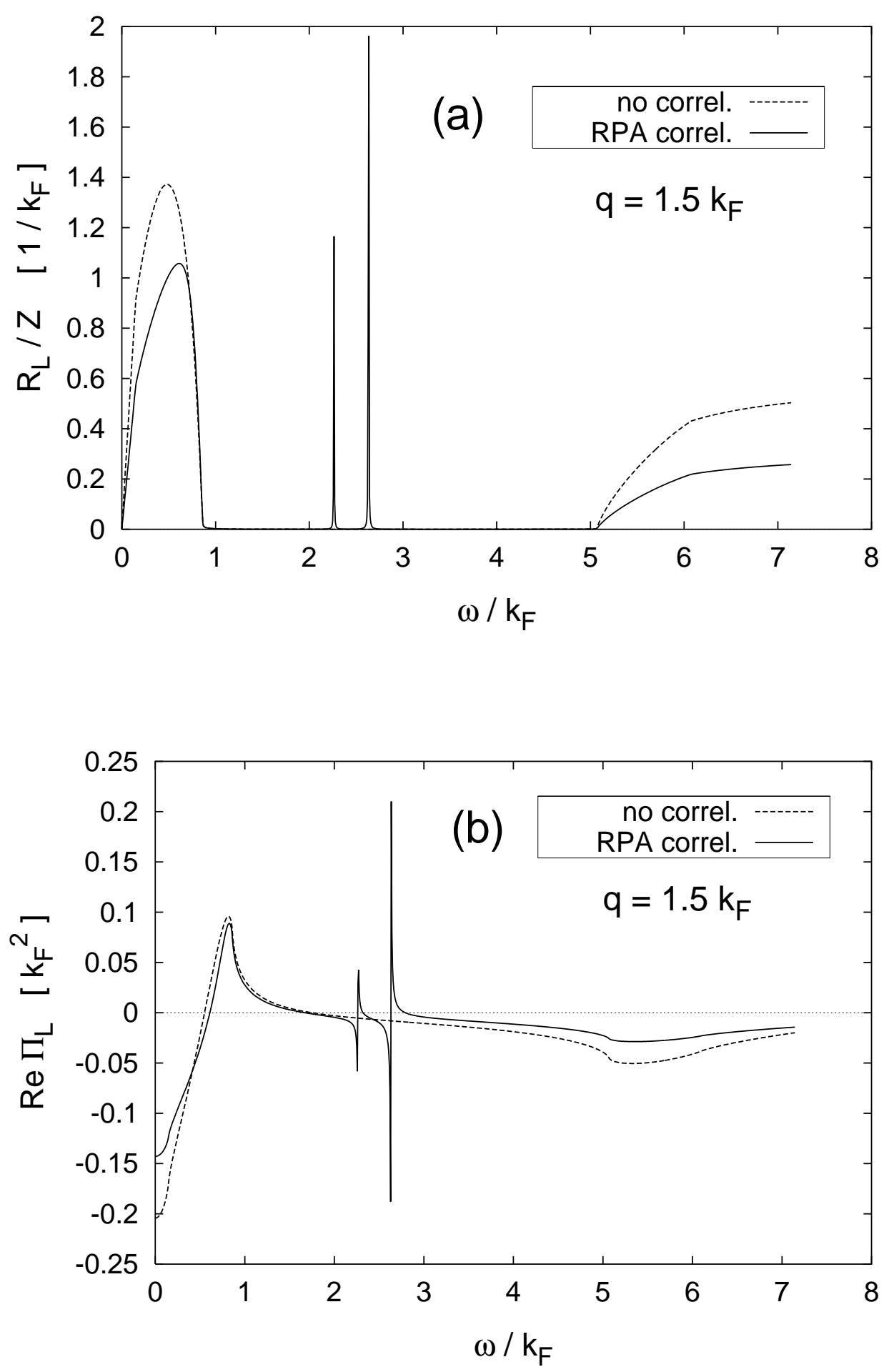

FIG. 3. Same as fig. 1 including vacuum polarization. 

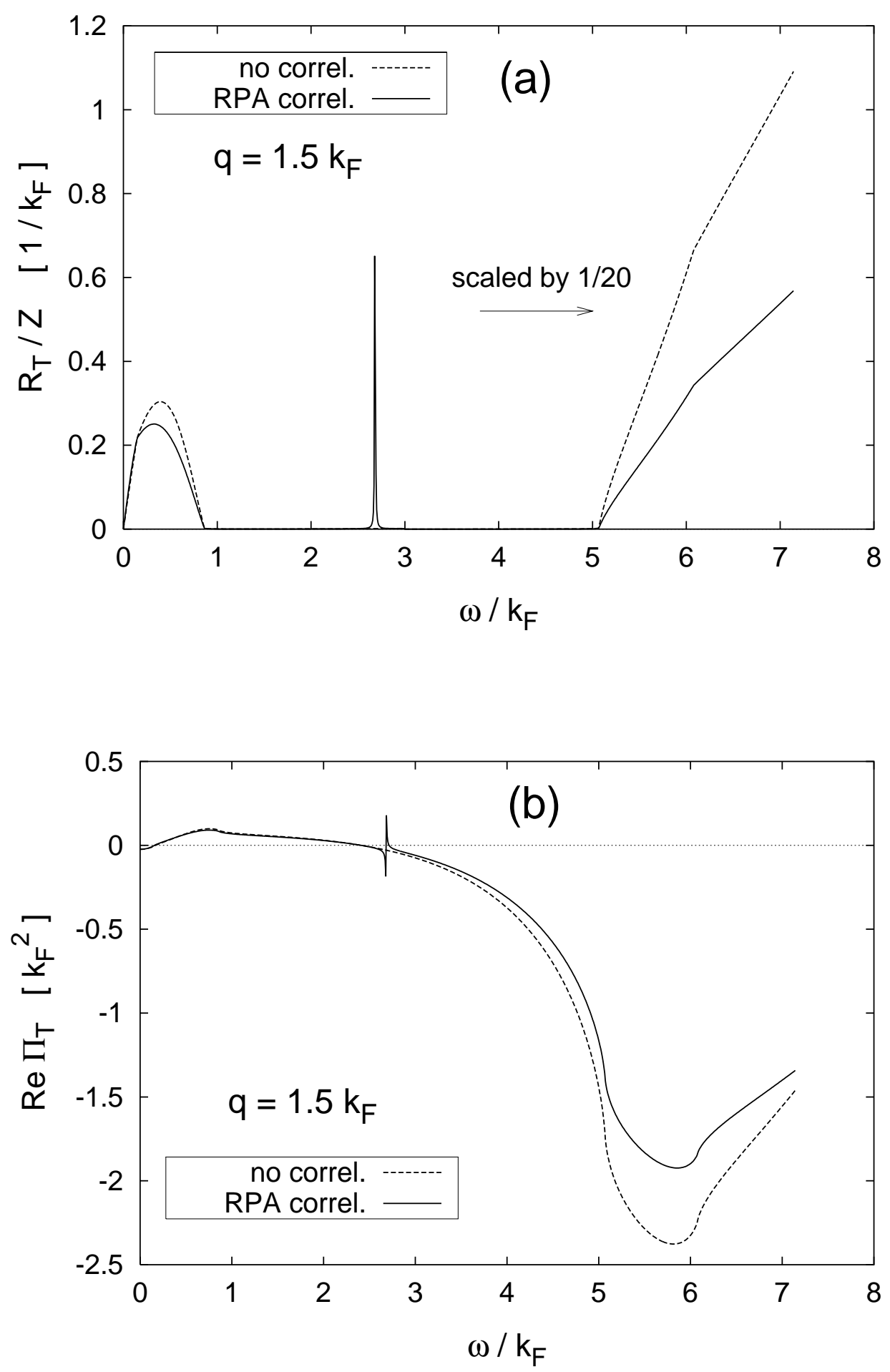

FIG. 4. Same as fig. 2 including vacuum polarization. Note that in (a) because of the fast increase of the transverse vacuum polarization this part of the curves is scaled by $1 / 20$. 

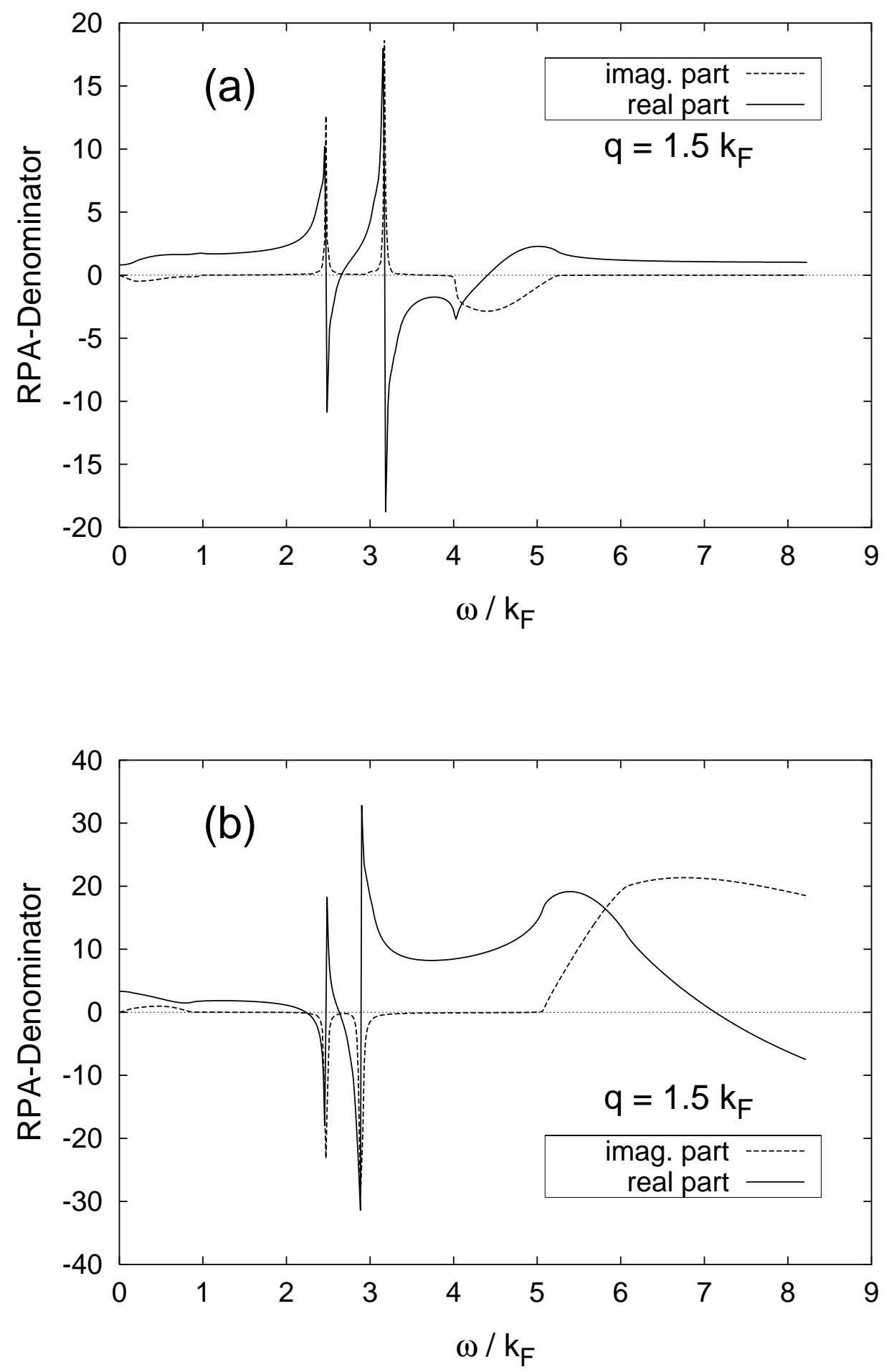

FIG. 5. Real part (full curves) and imaginary part (dashed) of the longitudinal RPA-denominator according to equ. (5). While in (a) vacuum polarization is neglected in (b) these contributions are included properly. 


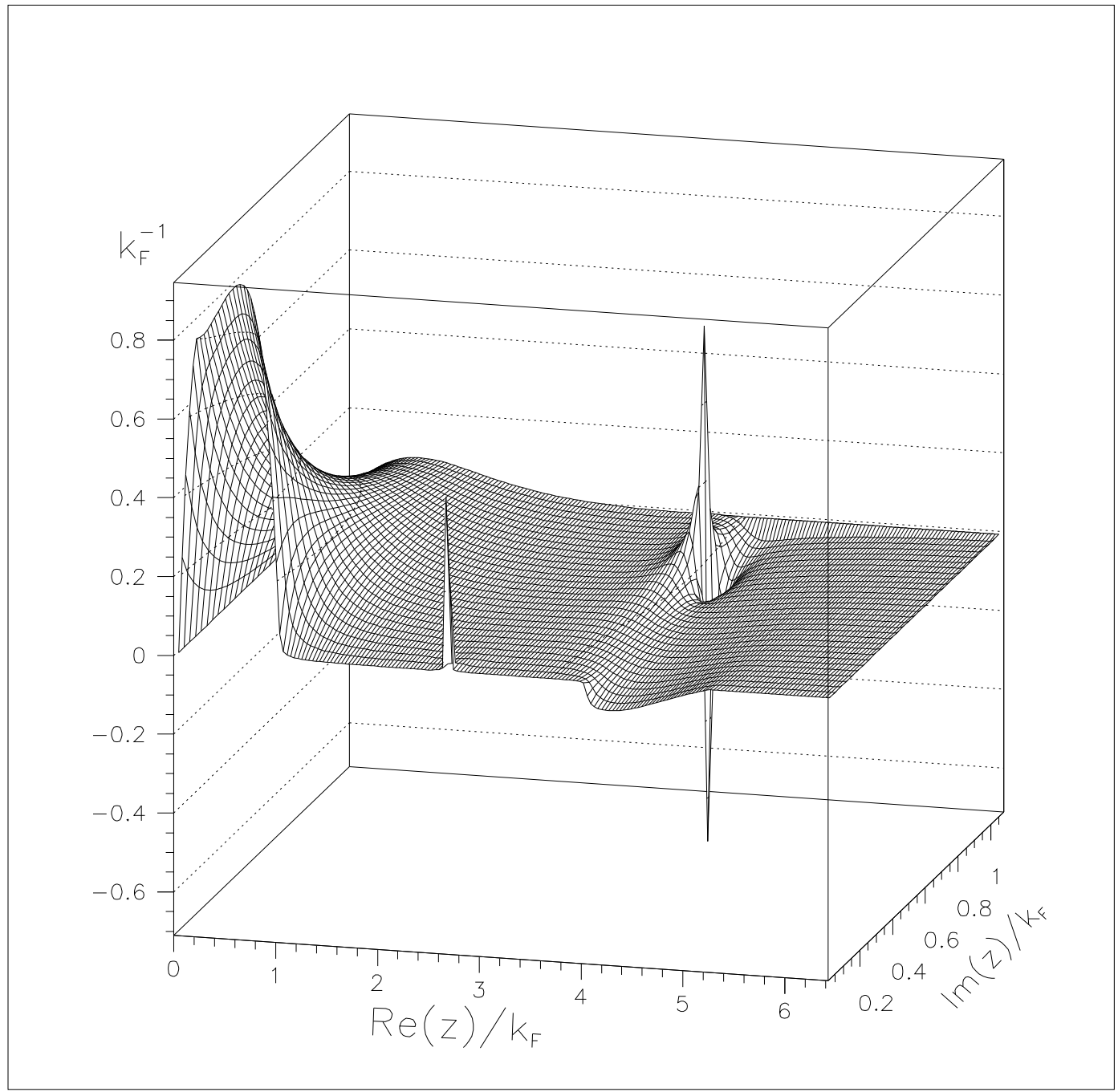

FIG. 6. Extension of the longitudinal RPA-response function to complex energies neglecting vacuum polarization (compare the full curve in fig. 1a). The big singularity in the upper complex energy plane is the signal for the violation of causality in this approximation. 


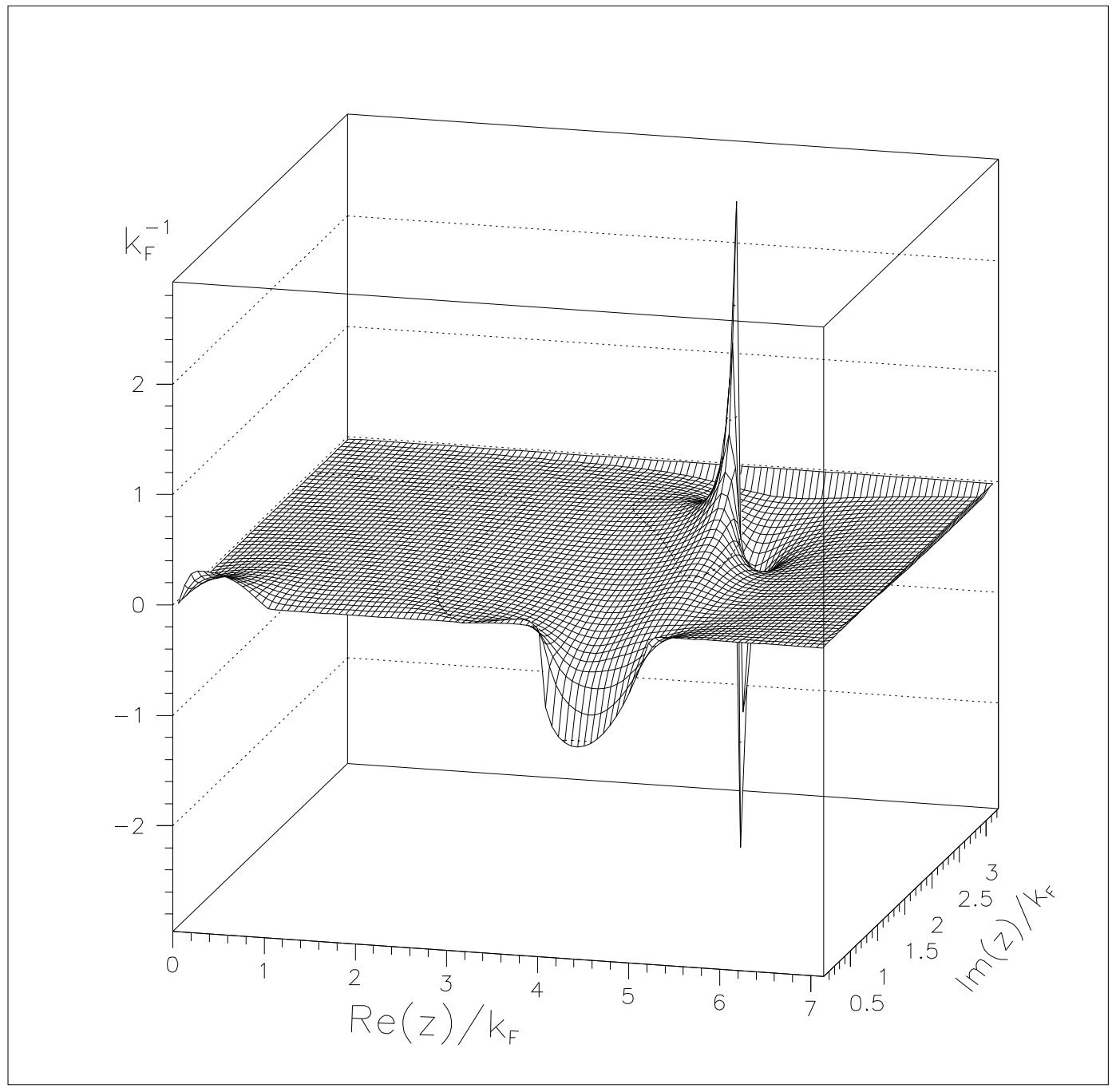

FIG. 7. Same as fig. 6 for the transverse response function. 